\title{
Interfacial amplification for graphene-based position-sensitive-detectors
}

\author{
Wen-Hui Wang ${ }^{1}$, Ru-Xia $\mathrm{Du}^{2}, \mathrm{Xi}-\mathrm{Tao} \mathrm{Guo}^{1}$, Jie Jiang ${ }^{1}$, Wei-Wei Zhao ${ }^{3}$, Zhong-Hua Ni ${ }^{3}$, Xin-Ran Wang ${ }^{4}$, \\ Yu-Meng You ${ }^{5}$ and Zhen-Hua $\mathrm{Ni}^{1}$
}

Light: Science \& Applications (2017) 6, e17113; doi:10.1038/Isa.2017.113; published online 20 October 2017

Position-sensitive-detectors (PSDs) based on lateral photoeffect have been widely used in diverse applications ${ }^{1-9}$, including optical engineering, aerospace and military fields. With increasing demand in long distance, low energy consumption, and weak signal sensing systems, the poor responsivity of conventional PSDs has become a bottleneck limiting their applications, for example, silicon $\mathrm{p}-\mathrm{n}$ or $\mathrm{p}-\mathbf{i}-\mathrm{n}$ junctions $\mathrm{s}^{2-5}$, or other materials and architectures ${ }^{6-10}$. Herein, we present a high-performance graphene-based PSDs with revolutionary interfacial amplification mechanism. Signal amplification in the order of $\sim 10^{4}$ has been demonstrated by utilizing the ultrahigh mobility of graphene and long lifetime of photo-induced carriers at the interface of $\mathrm{SiO}_{2} / \mathrm{Si}$. This would improve the detection limit of Si-based PSDs from $\mu \mathrm{W}$ to $\mathrm{nW}$ level, without sacrificing the spatial resolution and response speed. Such interfacial amplification mechanism is compatible with current Si technology and can be easily extended to other sensing systems ${ }^{11,12}$.

Figure 1a shows the representative schematic and the working principle of the graphene-based PSD, which contains a two-terminal graphene transistor deposited on the top of $300 \mathrm{~nm}$ thick $\mathrm{SiO}_{2}$ /lightly p-doped (1-10 $\Omega \mathrm{cm}) \mathrm{Si}$ substrate. The localized interface states ${ }^{13}$ such as positive charge states with energies within the silicon band gap exist at the oxide-silicon interface, induce a negative depletion layer $(-)$ in the silicon near the interface, making the surface energy bands bend downwards. This will lead to the formation of the intrinsic built-in electric field ${ }^{13,14}$ with the direction from the interface to bulk $\mathrm{Si}$, as shown in Figure 1b. Under the illumination of a point light source, as shown in Figure 1a, electron-hole pairs will be generated inside or within the minority-carrier diffusion length of the depletion region ${ }^{6,7}$, and then be separated by the built-in electric field. In such case, the photo-induced electrons would accumulate at $\mathrm{SiO}_{2} / \mathrm{Si}$ interface and diffuse laterally. When reach equilibrium, electrons will unevenly but steadily distribute at the interface, following a conventional lateral photoeffect-like behavior ${ }^{3-9}$. The electrons diffuse to the region under graphene will lead to an effective gate effect, consequent change the hole concentration and channel current through capacitive coupling. This would result in an ultrahigh gain or amplification, namely interfacial amplification ${ }^{14}$, which originates from the recirculation ${ }^{15}$ of holes in the graphene channel during the lifetime of electrons that accumulate at $\mathrm{SiO}_{2} / \mathrm{Si}$ interface. The interfacial amplification in our system is analog to photoconductive gain, which is determined by the ratio of lifetime and transit time of carriers between the two electrodes ${ }^{15}$. The gain ${ }^{16}$ or amplification is defined as $G=\tau_{l} / \tau_{t}$, here $\tau_{l}$ is the lifetime of photo-induced carrier at the $\mathrm{SiO}_{2} / \mathrm{Si}$ interface, $\tau_{t}$ is the transit time in the graphene channel. The extreme short transit time due to ultrahigh mobility of graphene ${ }^{17,18}$ and long lifetime of carriers at $\mathrm{SiO}_{2} / \mathrm{Si}$ interface would result in high gain $\mathrm{G}$ in our device. Such an interfacial amplification process behaves like a built-in amplifier, but it would not increase the noise level of the system (the signal noise in the graphene channel), so that the sensitivity of the device would be dramatically enhanced. The quantity of the accumulated electrons under the graphene channel varies with the incident light position, suggesting that different photocurrent will take place, and also the capability of ultrasensitive position detections of the light point by considering the high gain of interfacial amplification mechanism.

The position sensitive photoresponse characteristics of the device were recorded with laser focused on the device $(514 \mathrm{~nm}$, spot size $\sim 1 \mu \mathrm{m}$ ) under a fixed DC bias, $V_{\mathrm{DC}}=1 \mathrm{~V}$. Figure 1c shows typical photoresponse for our device, where the photo-switching characteristics of the graphene photodetector are plotted with light $(395 \mathrm{nW})$ incident on the substrate with different distances away from graphene channel. It is clear that considerable photocurrent generates and its magnitude declines significantly with increasing distance from the laser spot to graphene, as shown in Figure 1d. According to the nonequilibrium carrier diffusion theory, the amount of carriers decrease exponentially with the distance away from the laser spot due to

\footnotetext{
${ }^{1}$ State Key Laboratory of Bioelectronics, School of Physics, Southeast University, Nanjing 211189, China; ${ }^{2}$ Department of Basic Teaching, Nanjing Tech University Pujiang Institute, Nanjing 211134, China; ${ }^{3}$ Jiangsu Key Laboratory for Design and Fabrication of Micro-Nano Biomedical Instruments, School of Mechanical Engineering, Southeast University, Nanjing 211189, China; ${ }^{4}$ National Laboratory of Solid State Microstructures, School of Electronic Science and Engineering, and Collaborative Innovation Center of Advanced Microstructures, Nanjing University, Nanjing 210093, China and ${ }^{5}$ Ordered Matter Science Research Center, School of Chemistry and Chemical Engineering, Southeast University, Nanjing 211189, China

Correspondence: Z-H Ni, Email: zhni@seu.edu.cn

Received 12 February 2017; revised 21 July 2017; accepted 26 July 2017; accepted article preview online 30 September 2017
} 
a

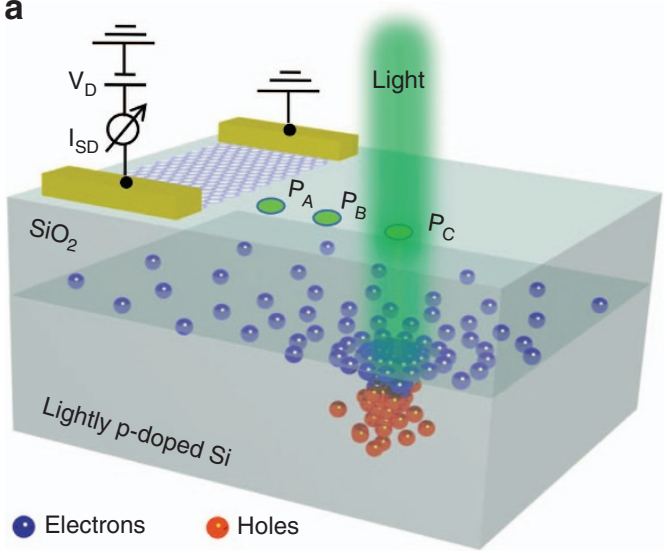

C

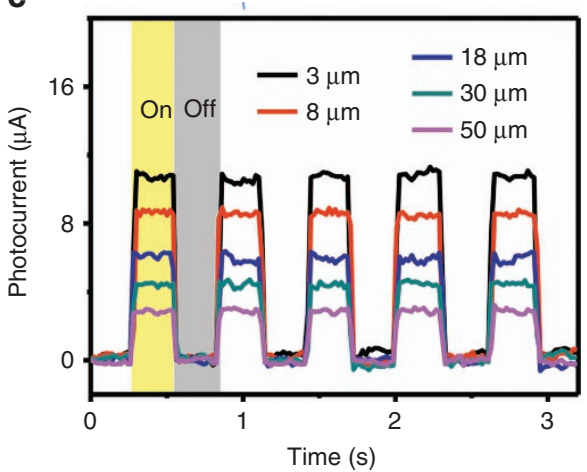

b

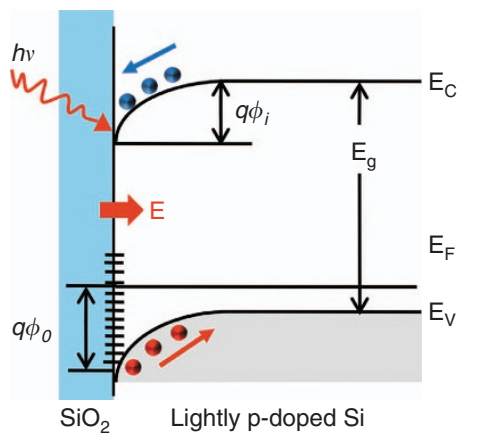

d

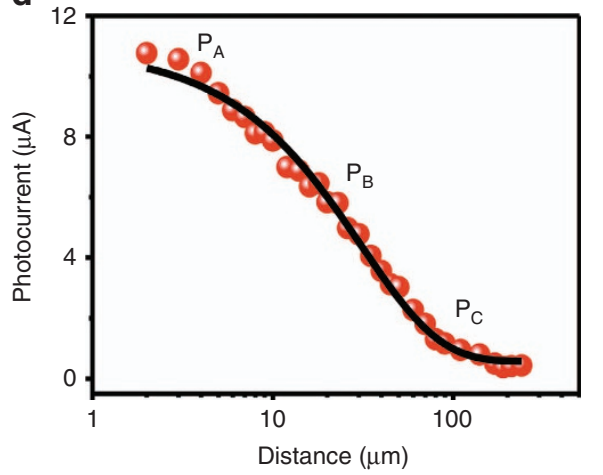

Figure 1 Schematic of graphene-based PSD with interfacial amplification. (a) Schematic diagram of the graphene-based PSD. Incident light creates electronhole pairs in the lightly p-doped silicon. Holes remain in the silicon, while electrons are accumulated at $\mathrm{SiO}_{2} / \mathrm{Si}$ interface and diffuse laterally. (b) Schematic of energy band diagrams of the lightly $\mathrm{p}$-doped silicon/ $\mathrm{SiO}_{2}$ interface with positive localized states. (c) Photo-switching characteristics of the graphene photodetector at different distance (from the laser spot to graphene device) using $395 \mathrm{nW}$ incident light. (d) The spatial dependence of the photocurrent. The black solid line is the exponential fitting curve of the experimental data.

recombination in the transport process, so does the photocurrent in the graphene channel. The exponential fitting curve in Figure 1d agrees well with the experimental photocurrent, confirming the mechanism of lateral photoeffect discussed above.

Figure $2 \mathrm{a}$ shows the position dependent photocurrent response of the PSD under different light power down to nW. We note that, even at extremely low incident power of $1.4 \mathrm{nW}$, a significant steady decrease of the photocurrent with the increasing distance is observed, suggesting excellent position sensitivity in our device to weak light signal. Furthermore, as seen in Figure $2 b$, a critical parameter in this system, the responsivity can be derived as high as $\sim 10^{3} \mathrm{~A} \mathrm{~W}^{-1}$. In conventional PSDs with silicon $(\mathrm{Si}) \mathrm{p}-\mathrm{n}$ or $\mathrm{p}-\mathrm{i}-\mathrm{n}$ junctions $\mathrm{s}^{2-5}$, photoexcited electron-hole (e-h) pairs are separated by built-in electric field existed at the junction, giving rise to a lateral potential gradient ${ }^{3}$ between the illuminated and non-illuminated zones, thus the photoinduced carriers will diffuse and are directly collected by two electrodes at both ends of surface layer. These devices are appealing for good linearity ${ }^{6-9}$ and fast response, but with relatively poor photoresponsivity $\left(<1 \mathrm{~A} \mathrm{~W}^{-1}\right)$. This is mainly because no effective gain mechanism exists in these structures. In our device, the interfacial amplification would result in a huge responsivity and improve the detection limit of PSDs from $\mu \mathrm{W}$ of conventional Si PSDs ${ }^{3-9}$ to $\mathrm{nW}$, demonstrating the potential of this device for weak signal detection. The laser spot size dependence of the photocurrent was also measured at $500 \mu \mathrm{m}$ away from graphene under different illumination intensity, as shown in Supplementary Fig. S3. The photocurrent keeps constant with variation of the spot size, in good accordance with the characteristic of 'independent of the incident light shape' of PSD. It should also be noted that the PSD can work in broad-band with wavelength range from 320 to $1100 \mathrm{~nm}$ as silicon is the photosensitive material.

Next, the time response of graphene-based PSD was studied by using an acoustic optical modulator with frequency of $10 \mathrm{kHz}$ to switch the light $(\sim 400 \mathrm{nW})$ on or off at $5 \mu \mathrm{m}$ away from the graphene channel. As shown in Figure $2 \mathrm{c}$, the rise $\left(\tau_{\mathrm{on}}\right)$ and fall $\left(\tau_{\text {off }}\right)$ time are $\sim 400 \mathrm{~ns}$ and $\sim 1.2 \mu$ s, respectively, where the rising and falling parts of the curves are fitted using a single exponential function. Since the recombination processes are statistically random, the photo-induced carrier lifetime $\tau_{l}$ at the interface is close to $\tau_{\text {off }}\left(10^{-6} \mathrm{~s}\right)$, while the transit time $\tau_{t}$ in the graphene channel is in the order of $10^{-10} \mathrm{~s}$ at $1 \mathrm{~V}$ bias for our device (the mobility is $\sim 8000 \mathrm{~cm}^{2} \mathrm{~V}^{-1} \mathrm{~s}^{-1}$ as shown in Supplementary Fig. S1(b)), leading to the amplification or gain $\left(G=\tau_{l} / \tau_{t}\right)$ of $\sim 10^{4}$. We have carried out control experiments by substituting high mobility graphene with low mobility $\mathrm{MoS}_{2}{ }^{19}$ (Supplementary Fig. S4), or substituting lightly doped $\mathrm{Si} / \mathrm{SiO}_{2}$ substrate with heavily doped $\mathrm{Si} / \mathrm{SiO}_{2}$ substrate (Supplementary Fig. S5), the photoresponse as well as gain is negligible. These results suggest that the ultrahigh mobility of graphene and the long lifetime of electrons at the interface of lightly doped $\mathrm{Si} / \mathrm{SiO}_{2}$ substrate are the two key factors for interfacial amplification.

The high speed response of the device could be attributed to the fast separation and diffusion of the photo-induced carriers at the lightly doped $\mathrm{Si} / \mathrm{SiO}_{2}$ interface. For better understanding of this kinetics, we 
a

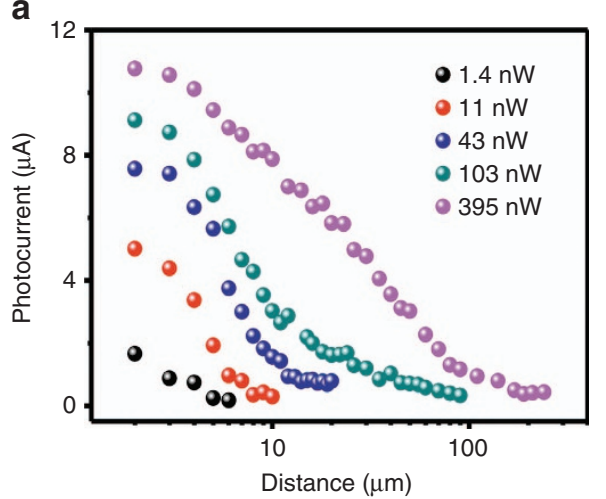

C

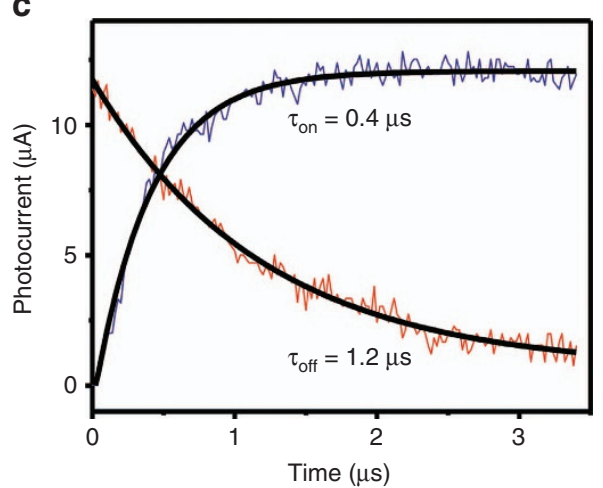

b

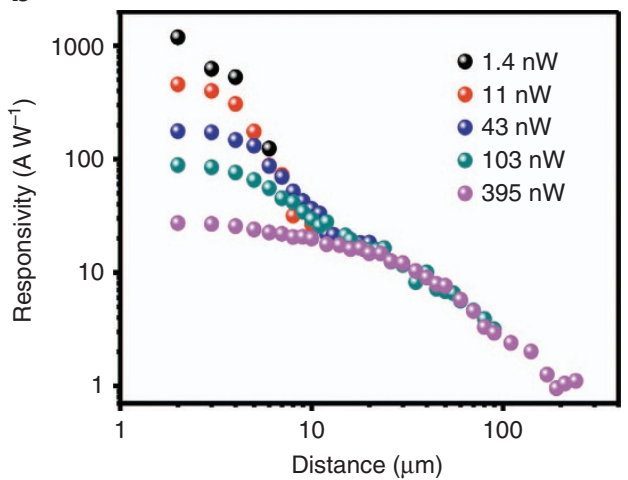

d

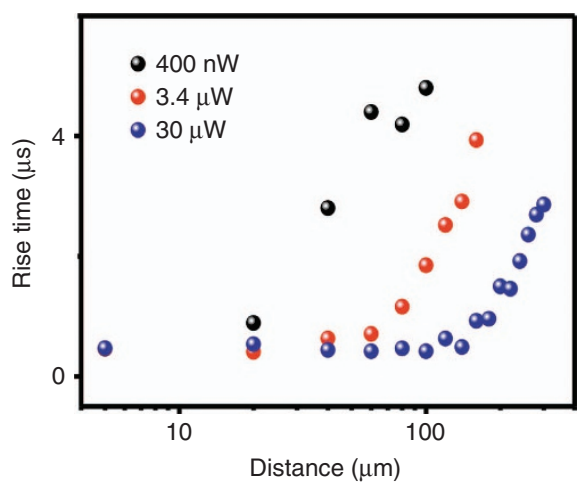

Figure 2 Performance of the graphene-based PSD. (a, b) Photocurrent and responsivity as a function of distance at $V_{D}=1 \mathrm{~V}$ under different weak light powers using $514 \mathrm{~nm}$ wavelength. (c) The transient response of the device at $5 \mu \mathrm{m}$ away from graphene, and light switched on or off by an acoustic optical modulator with frequency of $10 \mathrm{kHz}$. Light power $=\sim 400 \mathrm{nW}, V_{D}=1 \mathrm{~V}$. (d) The distance dependence of the rise time of the device under different illumination intensity.

analyzed the position dependent rise time with different illumination intensities, as shown in Figure $2 \mathrm{~d}$. The tested $\tau_{\text {on }}$ is almost flat and fixed on $\sim 500 \mathrm{~ns}$ within $20 \mu \mathrm{m}$ under $3.4 \mu \mathrm{W}, 100 \mu \mathrm{m}$ under $30 \mu \mathrm{W}$, indicating that the diffuse time of carriers in $\mathrm{Si} / \mathrm{SiO}_{2}$ interface or bulk $\mathrm{Si}$ is short and can be negligible within the corresponding distance ranges. However, when the distance excesses the threshold value, the diffusion time of carriers at the $\mathrm{SiO}_{2} / \mathrm{Si}$ interface can no longer be ignored and dramatically increase with distance. This can be explained by the change of concentration gradient of photo-induced carriers. Under low light intensity or the position far away from incident point, few photo-induced carriers exist at the interface and induce a small lateral potential gradient, which will increase diffusion time of the carriers. Despite the increase, the response speed of our PSD is still comparable with conventional Si-based PSDs, suggests that the interfacial amplification could greatly enhance the sensitivity of PSD without sacrificing the high response speed.

The kinetics of photo-induced carriers at the lightly p-doped Si/ $\mathrm{SiO}_{2}$ substrate was further characterized by using capacitance-voltage (C-V) measurements. For metal-insulator-semiconductor (MIS) structure, the capacitance equals to the capacitance of oxide layer and silicon surface space charge layer in series: $C=1 / C_{0}+1 / C_{s}$, where $C, C_{0}$ and $C_{s}$ are the measured oxide layer and silicon surface space charge layer capacitance, respectively. Figure $3 a$ displays the $\mathrm{C}-\mathrm{V}$ characteristic curves in dark and different illumination intensity at $5 \mathrm{kHz}$. It can be seen that the flatband voltage ${ }^{20}(\mathrm{VFB})$ is $\sim-4 \mathrm{~V}$, as the normalized flatband capacitance is $\sim 0.95$ for our substrate. This signifies that, under no bias, the energy band bend downwards at $\mathrm{Si}$ surface and there is a built-in electric field, in good agreement with the analysis shown in Figure 1b. The negative shift and increase of the capacitance minimum value under illumination suggest the injection of electrons at the interface of $\mathrm{SiO}_{2} / \mathrm{Si}$ with the increase of light power (more details about the $\mathrm{C}-\mathrm{V}$ measurements can be seen in Supplementary Fig. S6). Figure 3b presents the relationship between the photo-induced-specific capacitance $(\Delta C)$ and the illumination intensity at $V=0 \mathrm{~V}$. We found that the photo-induced-specific capacitance grows and finally saturates with the increase of incident power. It is because that the accumulated electrons progressively counteract the built-in electric field until completely balanced. The capacitance therefore reaches a steady state, which is in consistence with the saturation of photocurrent with the increase of incident power as mentioned above. In addition, we have also measured the capacitance of heavily doped silicon substrate, no response was found under the illumination. Hence, it is demonstrated that the lightly doped $\mathrm{Si} / \mathrm{SiO}_{2}$ interface plays a central role in the photosensitive behavior of our device.

To prove the capability of position detection of graphene-based PSD, a one-dimensional PSD was prepared by using two graphene devices $(\mathrm{S} 1, \mathrm{~S} 2)$ on the same substrate separated by $10 \mu \mathrm{m}$. Figure $4 \mathrm{a}$ shows position dependence of photoresponse of the two devices under $50 \mathrm{nW}$ incident light, and the optical image of the PSD is displayed in the inset of Figure 4a. The position sensitivity of the PSD can be observed from the increased or decreased photocurrent as incident light go toward or away from the graphene, and the position of incident light could be deduced by the photoresponse ratio $\left(I_{2}-I_{1}\right) /\left(I_{1}\right.$ $+I_{2}$ ) of the two devices, as shown in Figure $4 \mathrm{~b}$. It can be clearly seen that the ratio is zero at position near middle of the PSD, and performs 
a

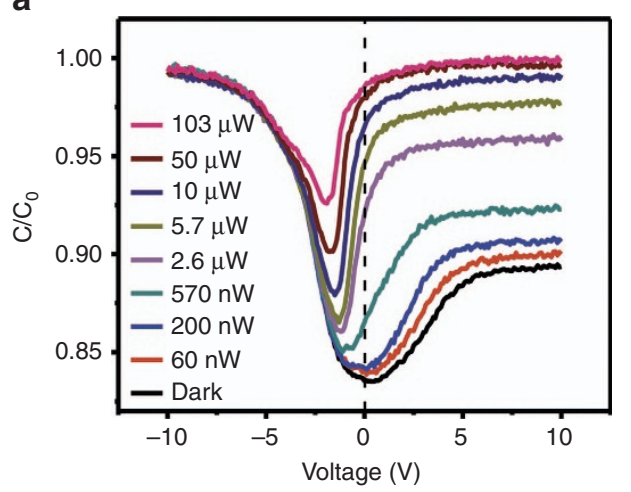

b

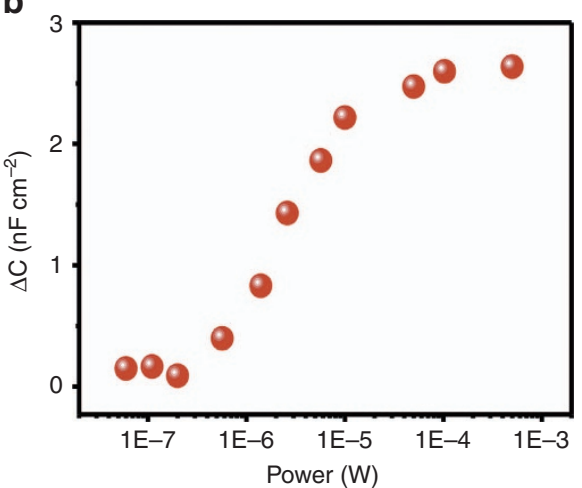

Figure 3 The capacitance characteristics of lightly $\mathrm{p}$-doped $\mathrm{SiO}_{2} / \mathrm{Si}$. (a) $\mathrm{C}-\mathrm{V}$ characteristic curves in dark and different illumination intensity at $5 \mathrm{kHz}$. (b) Power dependence of the photo-induced-specific capacitance at $V=0 \mathrm{~V}$.

a

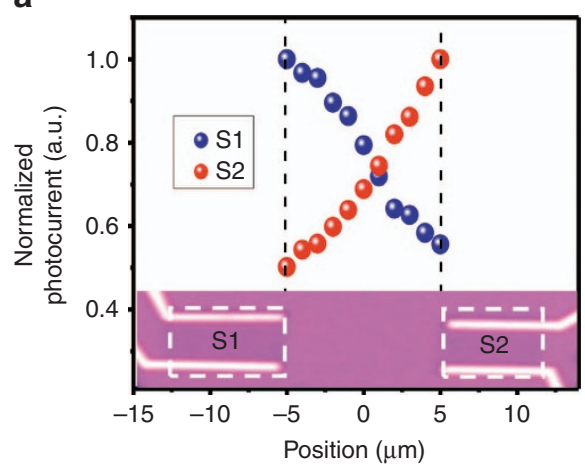

C

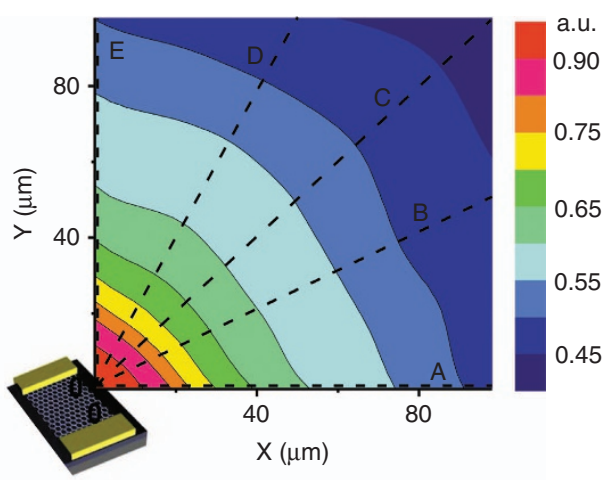

b

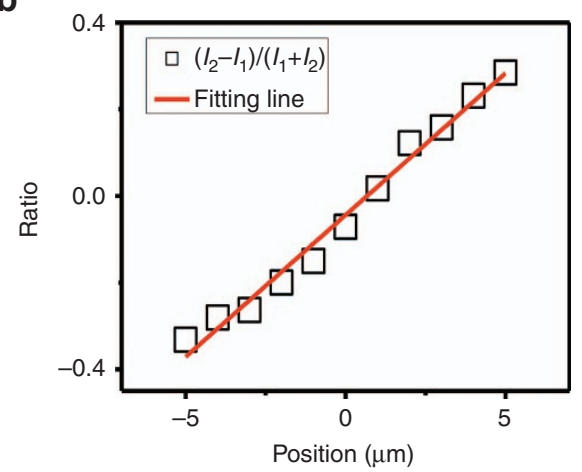

d

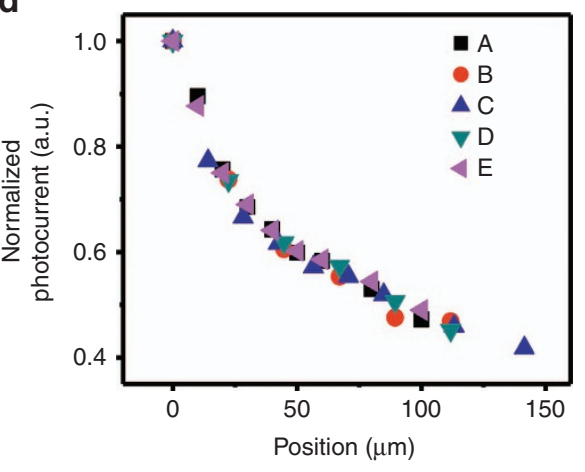

Figure 4 The representative models of the PSDs. (a) Position dependence of photoresponse of one-dimensional PSD prepared by using two graphene devices on the same substrate under $50 \mathrm{nW}$ incident light. The distance between two devices is $10 \mu \mathrm{m}$. Optical image of the PSD is displayed in the inset. (b) Photocurrent ratio $\left(I_{2}-I_{1}\right) /\left(I_{1}+I_{2}\right)$ of two devices as function of the position. (c) Two-dimensional spatial mapping of photocurrent of the graphene-based photodetector under $400 \mathrm{nW}$ incident light. (d) Photocurrent line scanning as labeled by directions $\mathrm{A}, \mathrm{B}, \mathrm{C}, \mathrm{D}, \mathrm{E}$ in (c).

well linear in detecting range. Considering the detection limit of photocurrent $(\sim 0.5 \mu \mathrm{A})$, the spatial position resolution should be less than $1 \mu \mathrm{m}$. Besides, two-dimensional spatial mapping of photocurrent under the illumination of $400 \mathrm{nW}$ laser is also demonstrated in Figure 4c. The overlapping distance dependence of photoresponse at different directions, as shown in Figure 4d, verifies that diffusion of the photo-induced carriers is isotropic. The uniform and protected $\mathrm{SiO}_{2} / \mathrm{Si}$ interface guarantees almost the same diffusion, which confirmed the potential utilization for ultrafast weak signal twodimensional PSDs.

The graphene-based PSDs reported in this work shows high responsivity and fast response time, which provides important advances and new development opportunity for future weak signal sensings.

\section{MATERIALS AND METHODS}

The single layer graphene sample is mechanically exfoliated from highly oriented pyrolytic graphite (HOGP) onto the top of $300 \mathrm{~nm}$ thick silicon oxide/lightly p-doped $(1-10 \Omega \mathrm{cm})$ silicon $\left(\mathrm{SiO}_{2} / \mathrm{Si}\right)$ substrate. The source and drain electrodes $(\mathrm{Ni}(5 \mathrm{~nm}) / \mathrm{Au}(50 \mathrm{~nm})$ ) were patterned by electron-beam lithography (FEI, FP2031/12 INSPECT F50), thermal evaporation (TPRE-Z20-IV), and lift-off processes. The optical image of the device as well as the Raman spectrum suggests the monolayer thickness of the graphene sample 
(Supplementary Fig. S1(a)). The transfer curve of the graphene device is also obtained and proves its high quality (Supplementary Fig. S1(b)). Photoresponse characteristics of the devices are measured using a Keithley 2612 analyzer. All the measurements were performed in air at room temperature. An Ar+ laser with wavelength of $\sim 514 \mathrm{~nm}$ is employed to attain the photocurrent response, which is focused on the sample with a $50 \mathrm{x}$ objective $(\mathrm{NA}=0.5)$ and the spot size of light is $\sim 1 \mu \mathrm{m}$. In the response time measurement, light was modulated with an acoustic optical modulator (R21080-1DS) at frequency of $10 \mathrm{kHz}$. A digital storage oscilloscope (Tektronix TDS 1012, $100 \mathrm{MHz} / 1 \mathrm{GS} / \mathrm{s}$ ) is used to measure the transient response of photocurrent. The photoresponse spatial scanning/mapping is obtained by moving the two dimensional stage and obtaining the photocurrent point by point. The $\mathrm{C}-\mathrm{V}$ characteristics of the substrate were measured by using Keithley 4200 semiconductor characterization system (Keithley, Cleveland, Ohio, USA).

\section{CONFLICT OF INTEREST}

The authors declare no conflict of interest.

\section{AUTHOR CONTRIBUTIONS}

ZHN conceived the project. WHW carried out device fabrication, electrical and photoresponse measurements and data analysis. XTG and JJ performed control device fabrications. WWZ and ZHN helped on the CV measurements. ZHN, WHW, RXD, XRW and YMY co-wrote the paper with all authors contributing to the discussion and preparation of the manuscript.

\section{ACKNOWLEDGEMENTS}

This work was supported by the National Key Research and Development Program of China (No. 2017YFA0205700), NSFC (61774034, 61422503 and 61376104), the Fundamental Research Funds for the Central Universities, and Research and Innovation Project for College Graduates of Jiangsu Province No. KYLX15_0111.

1 Arimoto S, Yamamoto $\mathrm{H}$, Ohno $\mathrm{H}$, Hasegawa $\mathrm{H}$. Hydrogenated amorphous silicon position sensitive detector. J Appl Phys 1984; 57: 4778-4782.

2 Lucovsky G. Photoeffects in nonuniformly irradiated $p-n$ junctions. J Appl Phys 1960; 31: 1088-1095

3 Fortunato E, Lavareda G, Vieira M, Martins R. Thin film position sensitive detector based on amorphous silicon $p-i-n$ diode. Rev Sci Instrum 1994; 65: 3784-3786.
4 Martins R, Fortunato E. Lateral photoeffect in large area one-dimensional thin-film position-sensitive detectors based in a-Si:H P-I-N devices. Rev Sci Instrum 1995; 66 2927-2934.

5 Vieira M. Speed photodetectors based on amorphous and microcrystalline silicon p-i-n devices. Appl Phys Lett 1997; 70: 220-222.

6 Levine BF, Willens RH, Bethea CG, Brasen D. Lateral photoeffect in thin amorphous superlattice films of $\mathrm{Si}$ and Ti grown on a Si substrate. Appl Phys Lett 1986; 49 1537-1539.

7 Xiao SQ, Wang H, Zhao ZC, Gu YZ, Xia YX et al. The Co-film-thickness dependent lateral photoeffect in $\mathrm{Co}_{-} \mathrm{SiO}_{2}$-Si metal-oxide- semiconductor structures. Opt Express 2008; 16: 3798-3806.

8 Henry J, Livingstone J. Optimizing the wavelength response in one-dimensional p-Si Schottky barrier optical PSDs. Phys Status Solidi A 2011; 208: 1718-1725.

9 Yu CQ, Wang H, Xia YX. Enhanced lateral photovoltaic effect in an improved oxidemetal-semiconductor structure of $\mathrm{TiO}_{2} / \mathrm{Ti} / \mathrm{Si}$. Appl Phys Lett 2009; 95: 263506.

10 Fontein PF, Hendrikst P, Woltert JH, Kucernakg A, Peat R et al. Differential measurements of the lateral photoeffect in GaAs/AIGaAs heterostructures. Semicond Sci Technol 1989; 4: 837-840.

11 Youngblood N, Chen C, Koester SJ, Li M. Waveguide-integrated black phosphorus photodetector with high responsivity and low dark current. Nat Photonics 2015; 9: 247-252.

12 Gan XT, Shiue RJ, Gao YD, Meric I, Heinz TF et al. Chip-integrated ultrafast graphene photodetector with high responsivity. Nat Photonics 2013; 7: 883-887.

13 Schroder DK. Surface voltage and surface photovoltage: History, theory and applications. Meas Sci Technol 2001; 12: R16-R31.

14 Guo XT, Wang WH, Nan HY, Yu YF, Jiang J et al. High-performance graphene photodetector using interfacial gating. Optica 2016; 3: 1066-1070.

15 Konstantatos G, Badioli M, Gaudreau L, Osmond J, Bernechea M et al. Hybrid graphene-quantum dot phototransistors with ultrahigh gain. Nat Nanotechnol 2012; 7: 363-368.

16 Liu YD, Wang FQ, Wang XM, Wang XZ, Flahaut E et al. Planar carbon nanotubegraphene hybrid films for high-performance broadband photodetectors. Nat Commun 2015; 6: 8589.

17 Novoselov KS, Geim AK, Morozov SV, Jiang D, Zhang Y et al. Electric field effect in atomically thin carbon films. Science 2004; 306: 666-669.

18 Novoselov KS, Geim AK, Morozov SV, Jiang D, Katsnelson MI et al. Two-dimensional gas of massless Dirac fermions in graphene. Nature 2005; 438: 197-200.

19 Radisavljevic B, Radenovic A, Brivio J, Giacometti V, Kis A. Single-layer $\mathrm{MoS}_{2}$ transistors. Nat Nanotechnol 2011; 6: 147-150.

20 Shyam R, Kulkarni DD, Field DA, Srinadhu ES, Harriss JE et al. Encapsulating Ion-solid interactions in metal-oxide-semiconductor (MOS) devices. IEEE Trans Nucl Sci 2015; 62: $3346-3352$.

cc)(1) (2) This work is licensed under a Creative Commons AttributionBY NC SA NonCommercial-ShareAlike 4.0 International License. The images or other third party material in this article are included in the article's Creative Commons license, unless indicated otherwise in the credit line; if the material is not included under the Creative Commons license, users will need to obtain permission from the license holder to reproduce the material. To view a copy of this license, visit http:// creativecommons.org/licenses/by-nc-sa/4.0/

(C) The Author(s) 2017

Supplementary Information for this article can be found on the Light: Science \& Applications' website (http://www.nature.com/lsa). 